\title{
ProtoEXIST: Advanced Prototype CZT Coded Aperture Telescopes for EXIST
}

\author{
Branden Allen*a, Jaesub Hong ${ }^{a}$, Josh Grindlay ${ }^{a}$, \\ Scott D. Barthelmy ${ }^{b}$, Robert G. Baker ${ }^{b}$, Neil A. Gehrels ${ }^{b}$, \\ Trey Garson ${ }^{c}$, Henric S. Krawwczynski ${ }^{c}$, \\ Walter R. Cook $^{d}$, Fiona A.Harrison ${ }^{d}$, \\ Jeffery A. Apple ${ }^{e}$, Brian D. Ramsey ${ }^{e}$ \\ ${ }^{a}$ Harvard-Smithsonian CfA, 60 Garden Street, Cambridge, MA 02138, USA \\ ${ }^{b}$ NASA Goddard Space Flight Center, Greenbelt, MD 20771, USA \\ ${ }^{c}$ Washington University, St. Louis, MO, 63130, USA \\ ${ }^{d}$ California Institute of Technology, Pasadena, CA 91125, USA \\ ${ }^{e}$ NASA Marshall Space Flight Center, VP62, Huntsville, AL 35812, USA
}

\begin{abstract}
ProtoEXIST1 is a pathfinder for the EXIST-HET, a coded aperture hard X-ray telescope with a $4.5 \mathrm{~m}^{2} \mathrm{CZT}$ detector plane a $90 \times 70$ degree field of view to be flown as the primary instrument on the EXIST mission and is intended to monitor the full sky every $3 \mathrm{~h}$ in an effort to locate GRBs and other high energy transients. ProtoEXIST1 consists of a $256 \mathrm{~cm}^{2}$ tiled CZT detector plane containing 4096 pixels composed of an $8 \times 8$ array of individual $1.95 \mathrm{~cm} \times 1.95 \mathrm{~cm} \times 0.5 \mathrm{~cm}$ CZT detector modules each with a $8 \times 8$ pixilated anode configured as a coded aperture telescope with a fully coded $10^{\circ} \times 10^{\circ}$ field of view employing passive side shielding and an active CsI anti-coincidence rear shield, recently completed its maiden flight out of Ft. Sumner, NM on the 9th of October 2009. During the duration of its 6 hour flight on-board calibration of the detector plane was

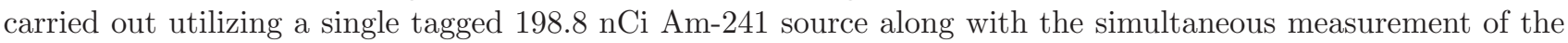
background spectrum and an observation of Cygnus X-1. Here we recount the events of the flight and report on the detector performance in a near space environment. We also briefly discuss ProtoEXIST2: the next stage of detector development which employs the NuSTAR ASIC enabling finer $(32 \times 32)$ anode pixilation. When completed ProtoEXIST2 will consist of a $256 \mathrm{~cm}^{2}$ tiled array and be flown simultaneously with the ProtoEXIST1 telescope.
\end{abstract}

Keywords: CZT, Hard X-Ray, Coded Aperture

\section{INTRODUCTION}

CZT (CdZnTe Cadmium-Zinc-Telluride) and CdTe (Cadmium-Telluide) is a semiconductor which has found increasing use in hard X-ray and soft $\gamma$-ray sensing applications over the past decade. The wide band gap $(\sim 2.5 \mathrm{eV})$ and high Z-composition make the detection of high energy X-rays / soft $\gamma$-rays $(\sim 5-2000 \mathrm{keV})$ possible at room temperature and eliminates the need for cryogenics which is traditionally required for other commonly utilized semiconductor detector materials such as high purity germanium (bandgap $\sim 0.62 \mathrm{eV}$ ) or silicon $(\sim 1.1 \mathrm{eV})$. For application in X-ray astronomy this removes a significant constraint to operation in a space environment through appreciable mass savings and eliminates the need for irreplaceable consumables increasing the upper bound of the mission lifetime. Long term implementation of high energy coded aperture telescopes utilizing CZT and CdTe detector planes have in fact been successfully demonstrated over the past decade in the INTEGRAL-IBIS ${ }^{\dagger}$ (Imager on Board INTEGRAL) using CdTe ${ }^{1,2}$ and the SWIFT-BAT (Burst $^{+}$ alert telescope) with CZT. ${ }^{3}$ The success of INTEGRAL and SWIFT has been encouraging and CZT/CdTe is

\footnotetext{
*Send correspondence to Branden Allen (ballen@cfa.harvard.edu)

${ }^{\dagger}$ Launch Date: $17^{\text {th }}$ October 2002 (Baikonur)

${ }^{\ddagger}$ Launch Date: $20^{\text {th }}$ November 2004 (Cape Canaveral)
}

Space Telescopes and Instrumentation 2010: Ultraviolet to Gamma Ray,

edited by Monique Arnaud, Stephen S. Murray, Tadayuki Takahashi, Proc. of SPIE Vol. 7732,

77324D · @ 2010 SPIE · CCC code: 0277-786X/10/\$18 - doi: 10.1117/12.857940

Proc. of SPIE Vol. 7732 77324D-1 
finding increased use in astronomy. In this first generation of space based detectors the individual pixels consisted of an individual detector crystal with a single readout. Advances in CZT detector technology have long since shown that the partitioning of a single detector into multiple detector pixels is possible by application of a pixilated anode without degradation in detector performance. ${ }^{4,5}$ This advance coupled with the introduction of new readout ASICs (application specific integrated chips) which consume less power-per-channel will enable the creation of even larger CZT detector planes at lower cost. Indeed such pixilated detectors have been flown in limited quantities as part of balloon payloads e.g. $\mathrm{HEFT}^{6}$ with a single detector and $\mathrm{InFOC} \mu \mathrm{S}^{7}$ with 4 detectors in a $2 \times 2$ configuration.

The ProtoEXIST program was conceived as a pathfinder for the development of an instrument with such a detector plane on a much larger scale: the EXIST-HET ${ }^{8,9}$ (high energy telescope on EXIST). The HET is currently envisioned to utilize $4.5 \mathrm{~m}^{2}$ of CZT consisting of $1126420 \mathrm{~mm} \times 20 \mathrm{~mm} \times 5 \mathrm{~mm}$ pixilated CZT detector units with $12 \times 10^{6}$ pixels total. Each detector units will have $32 \times 32$ pixels with a $600 \mu \mathrm{m}$ pixel pitch and consume $20 \mu \mathrm{W}$ per channel. In order to demonstrate the feasibility of such a detector plane using a scalable and highly redundant architecture the ProtoEXIST project has been divided into three phases:

1. ProtoEXIST1 (described here) is a coded-aperture telescope with a $256 \mathrm{~cm}^{2} \mathrm{CZT}$ detector plane tested in a space environment with a balloon flight at an altitude of $\sim 40 \mathrm{~km}$ above mean sea level. The detector plane utilizes the RADNET ASIC and is comprised of an $8 \times 8$ array of individual CZT detectors with pixilated anodes. Each individual detector had 64 pixels (a $8 \times 8$ array of pixels) or 4096 individual pixels over the entire detector plane. This phase culminated in a 6 hour flight out of Ft. Sumner, NM on the 9th of October 2009.

2. ProtoEXIST2 will demonstrate the feasibility of a nearly identical architecture utilizing the NuSTAR DBASIC (i.e. direct bond ASIC). ${ }^{6,10}$ This enables the demonstration of a detector plane with pixels possessing a $600 \mu \mathrm{m}$ pitch (i.e. a $32 \times 32$ pixel array for each detector) and fulfills the fine pixilization requirement of the EXIST-HET. The DB-ASIC currently consumes $80 \mu \mathrm{W}$ per channel exceeding the EXIST power requirement, necessitating the production of a new lower power version. Currently the design of the required detector electronics is nearing completion. At the time of this writing it is expected that testing of the first detectors of this series will begin within the next few months (i.e. October 2010).

3. ProtoEXIST3 will demonstrate the final unmet requirement for EXIST through a relatively minor modification of the DB-ASIC in order to produce the EX-ASIC (EXIST-ASIC) which will consume $20 \mu \mathrm{W}$ per channel. This phase of the program will conclude with a flight of the original detector module from ProtoEXIST1 and a second detector module consisting of 1/4 DB-ASIC mounted CZT detectors and 3/4 EX-ASIC mounted CZT detectors.

\section{PROTOEXIST1 PAYLOAD OVERVIEW}

The ProtoEXIST1 payload consists of two main parts: the gondola which supports flight operations and pointing functions, and the instrument package which contains all required subsystems for the support of the ProtoEXIST1 coded aperture telescope.

The main ProtoEXIST1 gondola (pictured in figure 1) was refurbished using the existing EXITE ${ }^{11}$ gondola frame which flew multiple times during the late 1980s and early 1990s. The pointing system as well as all supporting electronic subsystems were replaced using the $H_{E R O}{ }^{12}$ (High Energy Replicated Optics) gondola support electronics and pointing system design as a model. As in HERO the coarse azimuthal pointing system is based on a differential global positioning system (DGPS) unit with a backup magnetometer in place in the event of DGPS failure. A daytime star camera system (also based on a design from $H E R O^{13}$ ) and inertial guidance system are utilized for fine pointing control. The star camera has a $3^{\circ} \times 2^{\circ}$ field of view with a small pixel size $\left(3.5^{\prime \prime} \times 3.5^{\prime \prime}\right)$ which together with a long $(\sim 2.5 \mathrm{~m})$ baffle enables the resolution of stars over the daytime sky background. In addition two transmitters are mounted to the gondola, one for the transmission of reduced data to the ground for storage and analysis, and the other for the monitoring of the gondola and payload status. A single receiver is present for command and control and is linked to the standard command interface package 


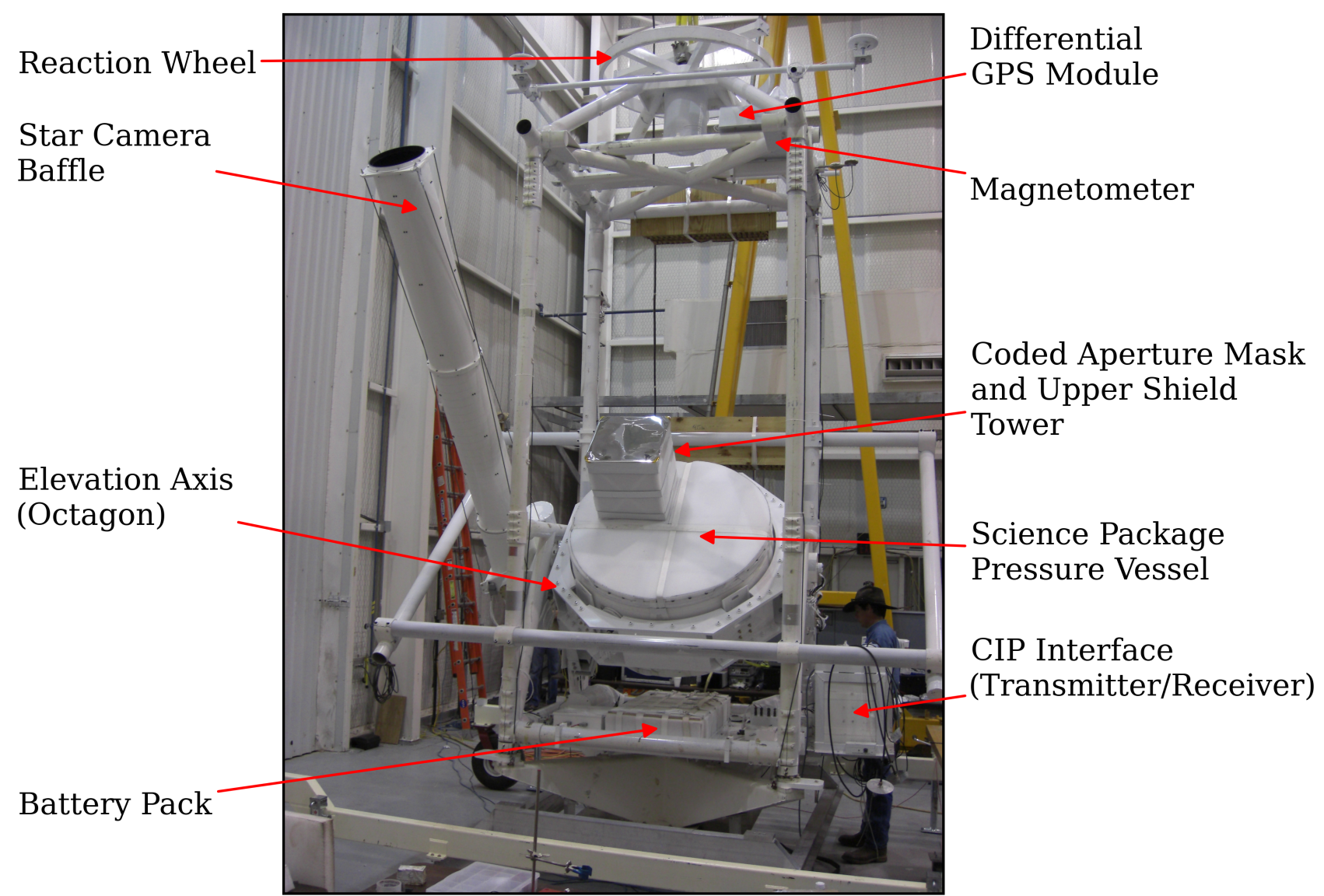

Figure 1: The integrated ProtoEXIST1 gondola 5 days prior to flight. The gondola supports the pointing, tracking, communications and timing subsystems and has an approximate height of $5 \mathrm{~m}$. The science package pressure vessel is directly mounted to the gondola through its octagonal support frame, which serves as the telescope's elevation axis. Visible to the left of the pressure vessel is the star camera used for independent aspect determination, which is mounted directly to the same elevation axis to preserve the boresight between the X-ray telescope and the star camera.

(CIP) provided for scientific balloon flights by the Columbia scientific balloon facility (CSBF) ${ }^{\dagger}$. The gondola also provides universal time (UT) synchronization information to the entire payload and experiment using a separate GPS receiver.

The remainder of the payload is contained within or mounted to a single $1.252 \mathrm{~m}$ diameter cylindrical aluminum pressure vessel with two removable end caps for payload access, and two removal self-sealing connector flanges which provide the electrical interface between the vessel and the gondola. For ProtoEXIST1 a single telescope consisting of:

1. the CZT detector plane $(\S 3)$

2. a lower (internal to the pressure vessel) side shield (graded Z-shield) (§4.1)

3. a upper side shield and mask support structure $(\S 4.1)$

4. the active CsI rear shield $(\S 4.2)$

5. a single $198.9 \mathrm{nCi}$ tagged ${ }^{241} \mathrm{Am}$ calibration source $(\S 4.2)$

\footnotetext{
${ }^{\dagger}$ http://www.csbf.nasa.gov
} 
Mask/Passive Shield Upper Tower

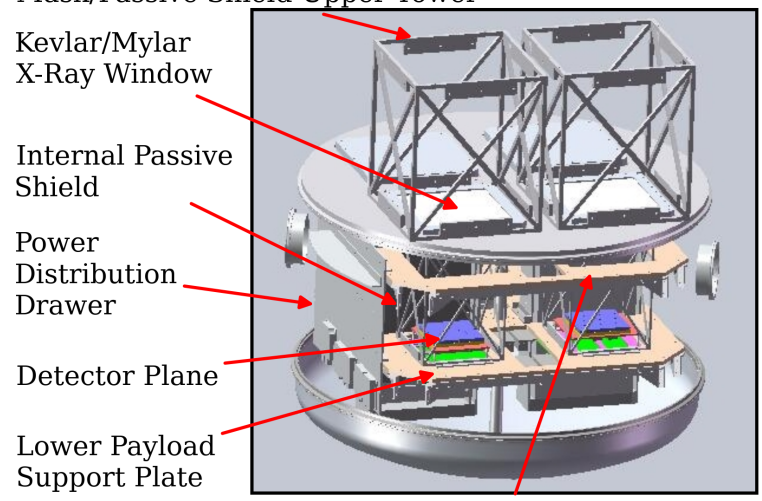

Upper Payload Support Plate
Power Distribution Drawer

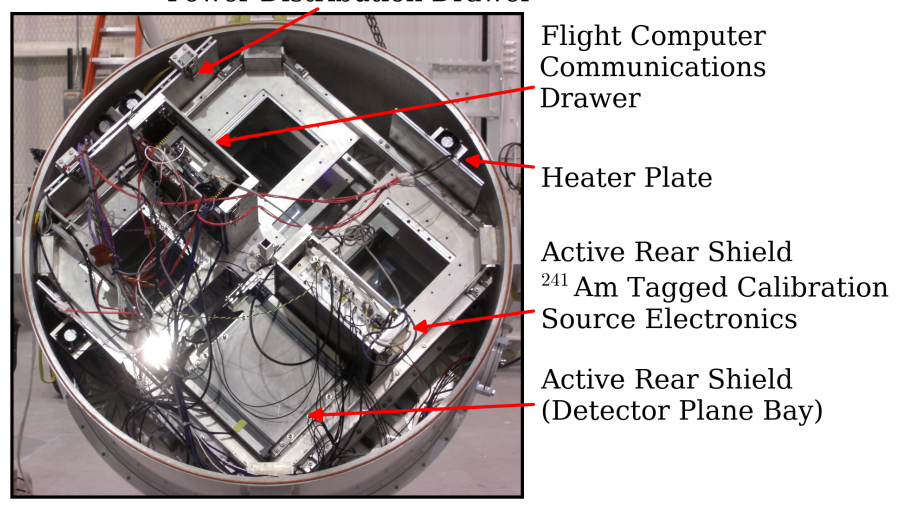

Figure 2: Left: A 3D model of the internal structure of the main science package pressure vessel. The vessel is shown here with two telescopes integrated into the payload, however only one full detector was produced and integrated for the flight. Right: a view of the rear of the fully integrate science package. The single integrated telescope sits directly above the active rear shield visible in the lower telescope port.

6. the coded aperture mask $(\S 4.4)$

is mounted in one of four available telescope slots, each of which is capable of accommodating a detector plane mounted within a EMI/RFI shielded enclosure with an approximate maximum size of $20 \mathrm{~cm} \times 20 \mathrm{~cm}$. The shielded enclosure has a thin $(6 \mathrm{mil}) \mathrm{Al}$ X-ray window for the observation of external sources. The internal structure of the pressure vessel (represented by the 3D model in figure 2) contains two main component mounting plates which are oriented perpendicular to the axis of the main vessel cylinder and are separated from one another by a distance of $288 \mathrm{~mm}$. The mounting plate located nearest to the bottom cap serves as the optical bench for the detector planes and as the mount point for the active rear shield (see $\S 4.2$ ). The top surface also contains mount points for the internal half of the passive side shielding (see §4.1) which surrounds the detector plane and runs up to the bottom surface of the top cap. The flat pressure vessel top cap is located approximately $400 \mathrm{~cm}$ above the top surface of the detector plane and is nominally $25.4 \mathrm{~mm}$ thick. The inner surface of the top cap has a honeycomb structure with a pocket depth of $14.48 \mathrm{~mm}$ to minimize distortion of the top cap due to internal pressure during flight while reducing the overall weight by about $30 \% .4$ ports are cut into the top cap onto which an airtight low mass X-ray window is mounted through which the detector plane is exposed to the overhead sky. The transparent region of the X-ray window assembly measures $28 \mathrm{~cm} \times 28 \mathrm{~cm}$ and is composed of a external $0.61 \mathrm{~mm}$ thick Kevlar layer and a single internal $0.12 \mathrm{~mm}$ thick mylar layer attached to an aluminum mounting frame. The Kevlar provides mechanical strength against the internal vessel pressure while the mylar provides the airtight seal. The thin, light design enables transmission of X-rays down to approximately $5 \mathrm{keV}$. Mounting points are located on the top surface of the pressure vessel top cap for the superstructure which supports coded aperture mask which in the final integrated payload is located $513 \mathrm{~mm}$ above the upper surface of the top cap.

Additional space within the vessel adjacent to the detector plane mounting ports serve as mount points for the science package electrical subsystems. There are eight additional ports in all: 4 centrally located between detector planes and 4 located in the area between the mounting plate and the outer vessel skin. In the current (ProtoEXIST1) configuration a flight computer, a transmitter interface card, 3 Ethernet controlled heater control relays, the active shield and tagged ${ }^{241} \mathrm{Am}$ calibration source control box, 2 primary pressure vessel heating elements, a power control, distribution, and monitoring box, and an Ethernet switch which serves as the hub of the science package internal communication system are mounted along with the prototype detector plane inside the vessel (see figure 2). The flight computer is the primary command/control and monitoring device for the science package and oversees all internal operations with the exception of the primary power control. The individual payload components communicate via an internal Ethernet network which serves as the primary communications link between the various subsystems. The flight computer is connected with a serial port (RS422 ) to the receiver on the gondola which serves as the command uplink for the science package. A command 
interpreter running continuously on the flight computer monitors the uplink for incoming commands and, when a command is received, determines the required action to be taken. The command is then (in most cases) forwarded across the internal network to the relevant subsystem. The flight computer is also linked to a transmitter interface card using a RS-422 interface and also acts as the primary downlink. The downlink is used to monitor the status of the instrument package and its associated subsystems as well as transmit reduced data packets from the detector plane to the ground. Each subsystem within the pressure vessel, including the detector plane, transmits status information over the network back to the flight computer where the downlink monitor, another persistent process running on the flight computer, monitors the incoming data, collates it and transmits all information back to the ground station. In order to prevent deadlocks the power monitor and distribution subsystem receives commands directly from the CIP interface, however status information is still sent via the flight computer to the downlink.

The entire science package is physically supported by the mounting plates which are coupled to 8 hard points embedded in the pressure vessel main body skin which in turn are attached directly to the octagonal support structure (visible surrounding the vessel in figure 1). This serves simultaneously as the main load bearing structure for the internal mechanical structure of the vessel and as the elevation axis for the coded-aperture telescope. The octagonal support structure mounts directly to the ProtoEXIST1 gondola via two elevation flanges which rotate on bearings affixed to the gondola superstructure. The elevation is monitored using a shaft angle encoder (SAE) located on the flange.

\section{THE PROTOEXIST1 DETECTOR PLANE}

The ProtoEXIST1 detector plane utilizes a modular architecture for redundancy and scalability. This design (as outlined section 1) is to be utilized for the construction of a much larger array i.e.EXIST. The ProtoEXIST1 detector plane consists of three primary components: a single flight control board (FCB), 8 detector crystal arrays (DCAs), 64 detector crystal units (DCUs). ${ }^{14-16}$

As shown in figure 3 each DCU consists of a $19.5 \mathrm{~mm} \times 19.5 \mathrm{~mm} \times 5 \mathrm{~mm}$ CZT crystal with a $8 \times 8$ pixilated anode bonded to a single interposer board (IPB) with single RadNet ASIC bonded to the side opposite the CZT. The CZT crystals are procured from Redlen technologies ${ }^{*}$ which pioneered the mass production of high quality low cost CZT using the traveling heater method (THM). CZT thusfar received have typically exhibited leakage currents below $0.6 \mathrm{nA}$ per pixel and are well within the operational specs of the ASIC (c.f. Allen et. al. in preparation $\left.{ }^{17}\right)$. The electrical and mechanical connection of the CZT is achieved using a low temperature solder bond ${ }^{\dagger}$ ( $\sim 40 \%$ of all ProtoEXIST1 detectors constructed) or transient liquid phase sintering (TLPS) bond ${ }^{\ddagger}$ between each CZT anode pixel and the corresponding IPB pad. The IPBs contains traces which route connections from the anode pads on the upper surface to output pads located on the underside of the IPB. The final connection of each pixel to the ASIC is made with a wirebond between the output pad and the ASIC input pad. A single square multi-pin connector is located on the underside of the ASIC which mates to a socket located on the upper side of the detector crystal array (DCA) upper board.

The DCA is composed of two printed circuit boards (PCB) with matching dimensions. Located on the upper surface of the upper board are $4 \times 2$ sockets to which individual DCUs are mounted to form a approximately $4 \mathrm{~cm} \times 8 \mathrm{~cm}\left(32 \mathrm{~cm}^{2}\right)$ detector module. The upper board also contains analog-to-digital converters (ADC) for the digitization of the ASIC sample readout as well as digital-to-analog converters (DAC) for the input of settings into the ASIC (i.e. thresholds, etc.). The bottom surface of the board supports two connectors which make the connection to the lower DCA board. The lower DCA board contains a single FPGA which handles the processing of data as well as command and control of each of the 8 DCUs mounted to the DCA. In order to reduce the number of required lines in the board the DCA FPGA handles 4 independent data channels each of which consists of the readout from 2 DCUs (a single DCU pair). Each DCU pair is effectively treated as a single detector except that threshold for each DCU may still be set independently. The bottom surface of the lower DCA board contains three surface mount connectors which interface to the flight control board (FCB).

\footnotetext{
${ }^{*}$ www.redlen.com

${ }^{\dagger}$ Low temperature solder bonding carried out at Delphon/QuikPak (http://www.delphon.com)

${ }^{\ddagger}$ TLPS bonding carried out at Creative Electron (http://www.creativeelectron.com)
} 

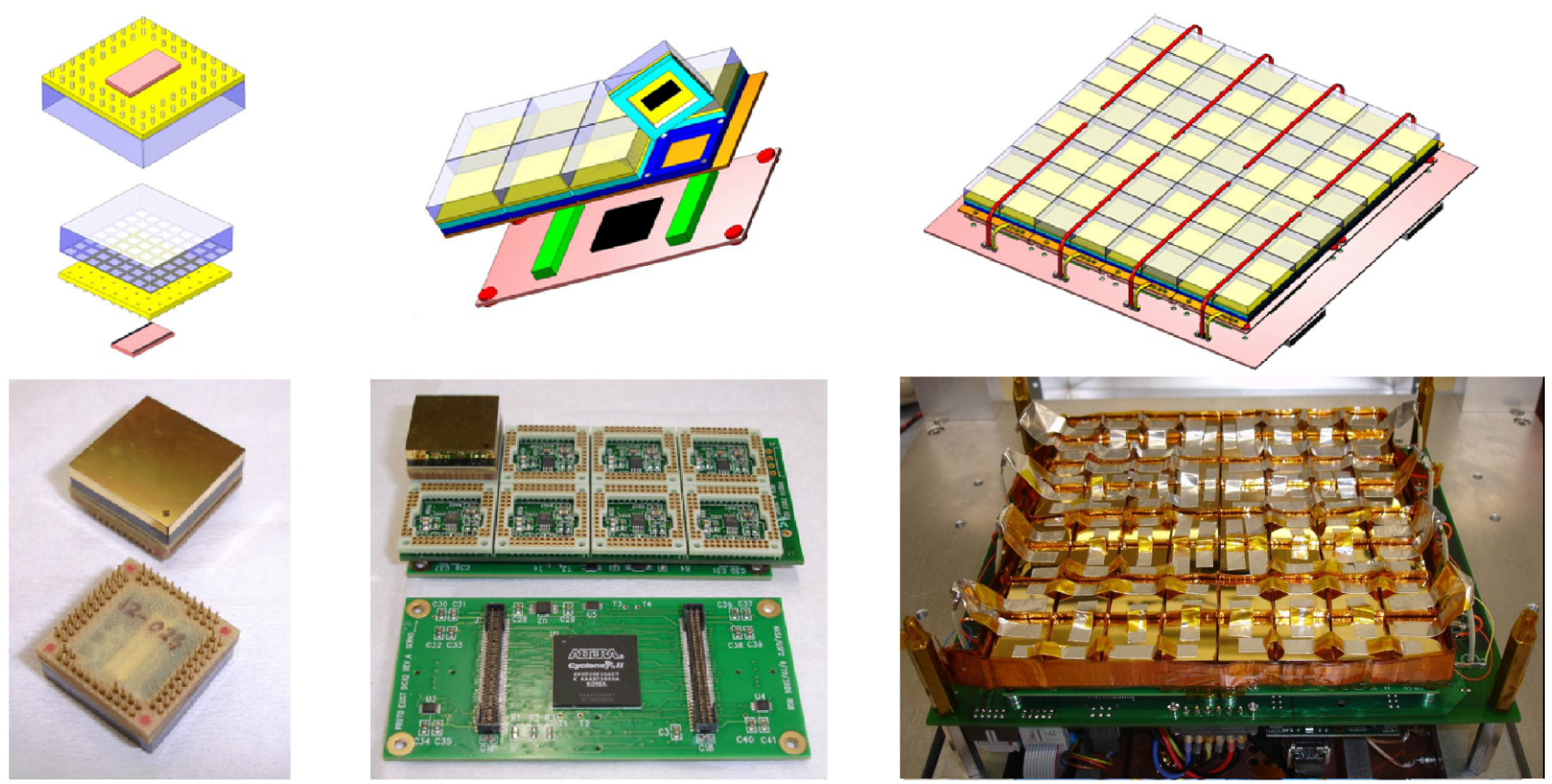

Figure 3: Left: A single $19.5 \mathrm{~mm} \times 19.5 \mathrm{~mm} \times 5 \mathrm{~mm}$ CZT crystal (shown in purple) bonded to the top surface of an interposer board (IPB, shown in yellow) constitutes a single detector crystal unit (DCU). The RadNet ASIC (shown in red) which reads out events and measures deposited charge on the CZT is bonded to the underside of the IPB. Center: A single detector crystal array (DCA) pictured with a single DCU mounted, supports a $4 \times 2$ array of DCUs $\left(\sim 32 \mathrm{~cm}^{2}\right)$. The lower DCA board contains a single FPGA which serves as the sole link for data transmission and command input for the mounted DCUs. Right: The fully integrated ProtoEXISTI detector plane with the detector module cover removed. A $2 \times 4$ array of DCAs $\left(\sim 256 \mathrm{~cm}^{2}\right)$ is mounted to the top surface of a flight control board (FCB) and constitutes a single complete detector plane. The individual detectors are coupled to a high voltage bias (nominally $-600 \mathrm{~V}$ ) through conductive Al tape (visible above) which are coupled to eight individual leads that are in turn connected to two high voltage power supplies located on the FCB.

The FCB supports a $2 \times 4$ array of DCAs (a total of 64 individual DCUs) which constitutes the complete $16 \mathrm{~cm} \times 16 \mathrm{~cm}\left(256 \mathrm{~cm}^{2}\right)$ detector plane. Two EMCO HVPSs are mounted to the underside the FCB, each with 4 leads (one per DCA) mounted on opposite sides of the detector plane. The HV leads and the cathodes of the individual DCUs are coupled with conductive $\mathrm{Al}$ tape (see figure 3). A thin flexible RFI/EMI shield consisting of a copper ground plane sandwiched between two layers of kapton tape surrounds the individual DCU to eliminate cross-talk between neighboring DCUs as well as reduce in the influence of any external sources of noise (c.f. Allen et. al. in preparation ${ }^{17}$ ). For handling of the data as well as command and control functions the FCB contains a single FPGA. All 32 (4 from each DCA) independent data channels are routed into the FCBFPGA and combined into a single data stream. The FPGA also serves as the terminal input point for timing, anti-coincidence, and calibration source signals. The FCB has 8 TTL inputs to accommodate discriminator output from the CsI rear shield, the ${ }^{241}$ Am tagged calibration source, and the 1 PPS time synchronization signal from the gondola GPS clock. In the ProtoEXIST1 flight configuration four of these inputs were utilized: two were required for each PMT on the CsI rear shield, and a single input was required for both the calibration source the 1 PPS GPS input. All of these signals are routed into the FCB FPGA where tagging and timing information may be applied to the event data as it is received. To ensure that the correct events are assigned the correct timing/tagging flags each of the 32 data channels from the detector pairs supports a fast trigger line, which connects directly to the FPGA. The fast trigger signals in combination with the external discriminator inputs are compared with an internally set $5 \mu$ s anti-coincidence window to identify background and calibration source events in the detector. After application of timing and tagging information the single data stream is 


\begin{tabular}{|c|c|c|}
\hline Shield Layer & Lower Shield $(\mathrm{mm})$ & Upper Sheild $(\mathrm{mm})$ \\
\hline $\mathrm{Pb}$ & $0.72(2.6)$ & $0.48(2.6)$ \\
$\mathrm{Sn}$ & $0.48(0.7)$ & $0.32(0.33)$ \\
$\mathrm{Cu}$ & $0.16(0.4)$ & $0.11(0.30)$ \\
\hline
\end{tabular}

Table 1: A list of the minimum required thickness for the individual layers of the ProtoEXIST1 graded Z-shield. The actual thickness of the final structures measured are given in parentheses.

sent from the FCB-FPGA to a Coldfire MCF5282 microcontroller ${ }^{\S}$ mounted on a Mod 5282 NetBurner card आ. The NetBurner card implements an Ethernet interface with the MCF5282 microcontroller and serves as the FCB's primary interface with the outside world. The MCF5282 serves 4 functions: the organization of the data stream into packets for transmission over Ethernet, the monitoring of the Ethernet port for user commands and the implementation of those commands on the proper subsystem, control of the high voltage supplies for the biasing detectors on the FCB, and the monitoring of the detector plane housekeeping data. The housekeeping data contains general status information on voltages, currents, and the board temperature which is combined into independent data packets and transmitted over the Ethernet. In ProtoEXIST1 all data and housekeeping packets are transmitted to the flight computer for storage on the payload data recorder (a solid state hard drive contained within the flight computer) and simultaneous downlink (see $\S 2$ )

\section{CODED APERTURE TELESCOPE}

The ProtoEXIST1 telescope occupies a single bay (out of a total of 4) in the pressure vessel and consists of four primary components: the graded Z-shield (§4.1) which restricts the detector plane's field of view to the coded aperture mask and partially shields the it from atmospheric X-ray albedo, the CsI active rear shield (§4.2) which enables the tagging atmospheric X-ray albedo events as well as the identification of cosmic ray background. The coded aperture mask (§4.4), a perfect binary uniform redundant array (URA) which is mounted 900 mm away from the cathode surface of the detector plane and enables the reconstruction of hard X-ray source positions, and the pixilated $16 \mathrm{~cm} \times 16 \mathrm{~cm}$ position sensitive detector plane $(\S 3)$ described above.

\subsection{Graded Z-Shield}

As previously alluded to the graded Z-shield is a passive shield installed in order to restrict the detector plane field of view to the coded aperture mask and to minimize the effects of the atmospheric albedo. The shield for a single telescope is divided into a $26 \mathrm{~cm} \times 26 \mathrm{~cm} \times 39.7 \mathrm{~cm}$ lower section, which sits inside the pressure vessel and surrounds the detector plane on 4 sides, and a $40 \mathrm{~cm} \times 40 \mathrm{~cm} \times 50 \mathrm{~cm}$ upper section which is affixed to the top cap of the science package pressure vessel. Both sections of the side shield are composed of laminated sheets of $\mathrm{Pb}-\mathrm{Sn}-\mathrm{Cu}$ (ordered from the outermost to the innermost layer) attached to an aluminum support frame. The design of the side shielding is based on the SWIFT-BAT graded Z-shield, ${ }^{18}$ however SWIFT-BAT employed a $\mathrm{Z}$-shield composed of $\mathrm{Pb}-\mathrm{Sn}-\mathrm{Ta}-\mathrm{Cu}$. Due to the prohibitive cost of $\mathrm{Ta}$ at the time of construction this layer was dropped from the ProtoEXIST1 design and the thickness of the $\mathrm{Sn}$ and $\mathrm{Cu}$ layers were increased in order to compensate for its removal. The inner layers of the graded Z-shield serve to attenuate the $\mathrm{Pb}$ fluorescence lines and, similarly, the innermost $\mathrm{Cu}$ layer attenuates the remaining Sn fluorescence lines, the Cu fluorescence lines $(\sim 8 \mathrm{keV})$ lie below the minimum detector threshold. Due to the discovery of a minor mechanical problem with the internal vessel structure a rebuild of the lower section of the side shield was required. As a result a portion of the $\mathrm{Pb}$ layer at the top of the lower section approximately $30 \mathrm{~cm}$ distant from the detector plane surface was left unshielded due the lack of additional $\mathrm{Sn}$ and $\mathrm{Cu}$ in the field. As a result the $\mathrm{Pb}-\mathrm{K} \alpha$ and $-\mathrm{K} \beta$ florescence lines were observed emanating from the exposed section during the flight (see §5).

\subsection{Active Rear Shielding and ${ }^{241}$ Am Calibration Source}

The shield electronics box is the subsystem/module that is responsible for the readout of raw PMT signals from the active rear shield and the ${ }^{241} \mathrm{Am}$ calibration source. For this purpose the shield electronics box contains

\footnotetext{
$\S$ http://www.freescale.com

『 http://www.netburner.com/products/core_modules/mod5282.html
} 
HV outputs for the biasing of PMTs, and computer controlled discriminator thresholds and outputs. The shield system is controlled by the flight computer over a serial port. A connection to the internal Ethernet network is available and used to send raw PMT pulse rates for both the CsI rear shield and the ${ }^{241} \mathrm{Am}$ source. The discriminator outputs for each PMT are linked directly to the detector plane for anti-coincidence timing. The active rear shield itself is composed of a single $26 \mathrm{~cm} \times 26 \mathrm{~cm} \times 2 \mathrm{~cm}$ CsI crystal coupled to two PMTs across two wave shifter bars mounted on opposite sides of the scintillator. The PMTs are monitored individually and have separate discriminator output lines. The calibration source consists of a small aluminium cylinder that is filled with a ${ }^{241} \mathrm{Am}$ doped scintillator. A clear window to which a single PMT is optically coupled is located at the top end of the cylinder which enables the detection $5.47 \mathrm{MeV} \alpha$-particles which are emitted in coincidence with the $59.6 \mathrm{keV}$ X-rays used for detector calibration. The calibration source PMT has an independent discriminator input/output line on the shield electronics box as well that enables the identification or tagging of calibration $\mathrm{X}$-ray events in the detector plane. The calibration source tagging efficiency observed in the hanger and during the flight is approximately $75 \%$.

\subsection{Detector Integration and Performance}

The integration of the ProtoEXIST1 proceeded in two steps. Newly bonded DCUs were received at a rate of about 8-10 per week during peak production and integrated onto a single DCA for preliminary testing. Due to the production rate and time constraints most DCUs were not tested individually but tested together in a fully populated DCA. Previous tests have not revealed a significant difference in performance for healthy detectors operating in an array provided that the EMI/RFI shielding completely surrounds each DCU and is properly grounded. The test array would then undergo a short pulser test at $0 \mathrm{~V}$ for the detection of dead pixels, a pulser test at $600 \mathrm{~V}$ for the detection of hot pixels and any other possible anomalies, and finally undergo a ${ }^{57} \mathrm{Co}$ calibration test. Detectors with more than 3 dead pixels or other anomalies were replaced and the DCA retested as needed. Once a good candidate DCA was completed it was set aside for integration into the flight detector.

The flight detector was then integrated $1 \mathrm{DCA}$ at a time. After the installation a single DCA the $0 \mathrm{~V}$ pulser, $600 \mathrm{~V}$ pulser, and ${ }^{57} \mathrm{Co}$ calibration tests were repeated on the partially completed array to detect any changes with the addition of a single DCA. This was repeated until the installation of all 8 fully populated DCAs was complete. During the detector integration and after arrival in the field at Ft. Sumner some detectors $\sim 5$ developed anomalies and required replacement.

During each integration step, with the exception of a few anomalous detectors which went bad during the course of pre-flight testing, no substantial changes in detector performance were observed between the operation individual isolated DCUs, DCUs integrated into a single $2 \times 4$ array on a DCA, and DCAs integrated into the array of $2 \times 4$ DCAs $(64 \times 64$ DCUs). In each case the detectors maintained an average resolution of $3.5 \mathrm{keV}$ (FWHM) at $59.6 \mathrm{keV}$. A more complete account will be available in Allen et. al. ${ }^{17}$

\subsection{Coded Aperture Mask and Imaging Performance}

The ProtoEXIST1 telescope utilizes a $64 \times 64$ element prefect binary uniform redundant array (URA) (c.f. references ${ }^{19,20}$ ) placed $900 \mathrm{~mm}$ from the surface of the detector plane for image reconstruction. $4.2 \mathrm{~mm}$ square pixels with a pitch of $4.7 \mathrm{~mm}$ are utilized for the mask pattern, the support grid (i.e. the material for support of the mask structure running between pixels) has a thickness of $0.5 \mathrm{~mm}$. The mask is composed of 12 identical laminated tungsten sheets into which the URA pattern was chemically etched ${ }^{*}$. The assembled mask is mounted into an aluminium frame which sits atop the outer side shield tower on the upper surface of the ProtoEXIST1 pressure vessel. Due to the difference between the coefficients of thermal expansion for $\mathrm{Al}$ and $\mathrm{W}\left(\alpha_{\mathrm{Al}} / \alpha_{\mathrm{W}} \sim 5\right)$ and the wide range of temperatures experienced in the upper atmosphere (between $-70^{\circ} \mathrm{C}$ and $20^{\circ} \mathrm{C}$ ), the mask is coupled to the mounting frame via a Si rubber gasket in order to prevent damage or warping of the mask pattern as the frame expands and contracts during the flight. The mask also has 8 hard attachment points (at 1 each corner and 1 at each midpoint) buffered by a gasket to ensure that the mask remains in the same position relative to the detector plane during the flight. Four additional $3 \mathrm{~mm}$ diameter holes are etched into each pattern and are used for the attachment of alignment pins which prevent the mask layers from slipping out of alignment. The final assembled telescope has a $10^{\circ} \times 10^{\circ}$ fully coded field of view and an angular resolution of $21^{\prime}$.

\footnotetext{
*Photo-etched at Tech-Etch, Plymouth, MA http://www.tech-etch.com
} 


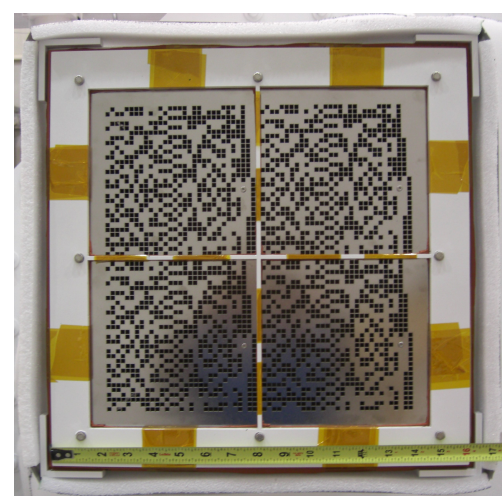

(a)

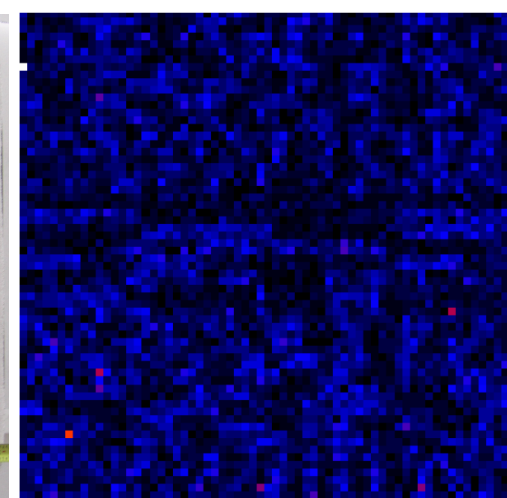

(b)

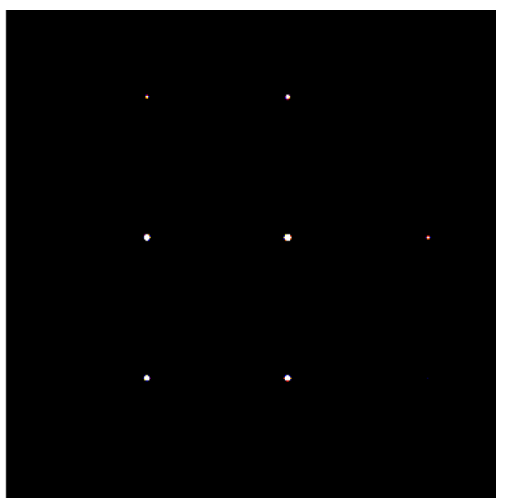

(c)

Figure 4: (a) The ProtoEXIST1 URA mask and support tower mounted to the top surface of the pressure vessel prior to flight. Each pixel is $4.2 \mathrm{~mm}$ square and is separated by a $0.5 \mathrm{~mm}$ thick support grid. Prior to flight an imaging test was carried out using a $3 \mathrm{mCi}{ }^{57} \mathrm{Co}$ source placed approximately $3 \mathrm{~m}$ from the surface of the mask. (b) The mask pattern can be seen in the detector plane image generated from the data collected during the imaging test. (c) The reconstructed image produced from a FFT cross correlation between the mask pattern and the raw detector image clearly reconstructs the source. Due to the cyclic nature of the URA 8 "ghost" images are also visible.

Prior to the construction of the mask a series of imaging simulations were carried out for optimization of the mask pattern. 10 separate masks were simulated utilizing the current URA pattern with different scale sizes between $4.5 \mathrm{~mm}$ and $5.5 \mathrm{~mm}$ at equal $0.1 \mathrm{~mm}$ steps within this range. In each case the $0.5 \mathrm{~mm}$ support grid was retained and the mask pixel size was reduced to achieve the change in pixel pitch. The detector plane was modeled to represent the real detector plane as closely as possible with $600 \mu \mathrm{m}$ gaps between each DCU. Due to the structure of the detector plane and mask additional induced coding noise was predicted to occur in detector images generated with the planned $5.0 \mathrm{~mm}$ mask. From these simulations it was determined that a mask with a $4.7 \mathrm{~mm}$ pitch minimized this effect and was ultimately adopted for use in the ProtoEXIST1 telescope. An imaging verification test was carried out with the fully integrated telescope prior to flight utilizing a single 3 $\mathrm{mCi}{ }^{57} \mathrm{Co}$ source placed at an approximate distance of $3.0 \mathrm{~m}$ from the surface of the mask. The performance of the test image was consistent with that of the simulated images after taking the magnification factor due to the placement of the source at a finite distance into account (Allen et. al. in preparation ${ }^{17}$ ).

\section{FLIGHT PERFORMANCE}

ProtoEXIST1 began its maiden flight at 14:40 UT on the $9^{\text {th }}$ of October 2009. The gondola reached float altitude (nominally $39 \mathrm{~km}$ ) approximately 1.5 hours after liftoff (see figure 5 a and table 2). The observation plan was initiated with an attempted observation of the quasar 3C 273, however during the first half of the flight due to instability in the elevation axis a stable pointing count not be achieved, presumably due to a stiff wire on the elevation axis. After the end of the 3C 273 observation run testing of the CsI rear shield thresholds was carried out over a period of approximately $30 \mathrm{~min}$. At 15:00 an observation of the black hole binary Cyg X-1 was initiated and carried out over the course of one hour. A short system power control test and detector restart was initiated after which time the Cyg X-1 observing run was re-initiated until flight termination. Cyg X-1 was successfully observed and the payload was recovered with minimal damage to the gondola. The science package was recovered unharmed.

In spite of the short duration of the flight and the additional difficulties encountered with the pointing system the primary objectives: the detection of a celestial source (Cyg X-1) with the ProtoEXIST1 coded aperture telescope, the successful operation of the detector plane and measurement of backgrounds in a near space environment, and verification that all the payload subsystems functioned as designed in flight were all accomplished. 


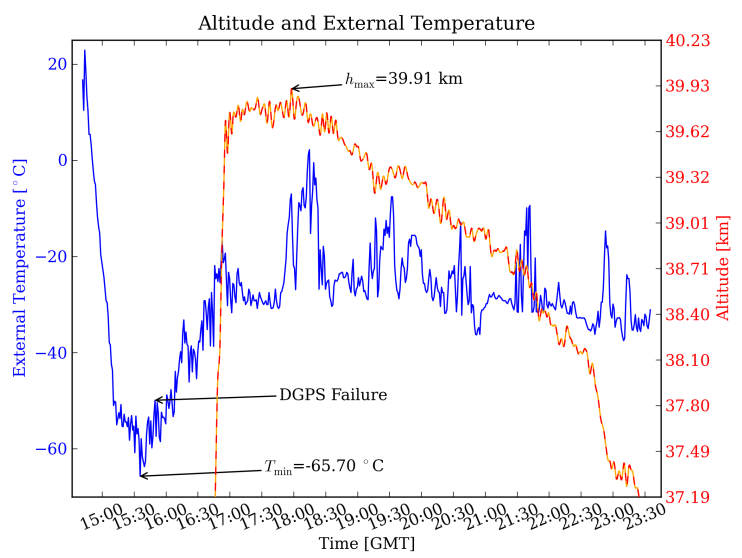

(a)

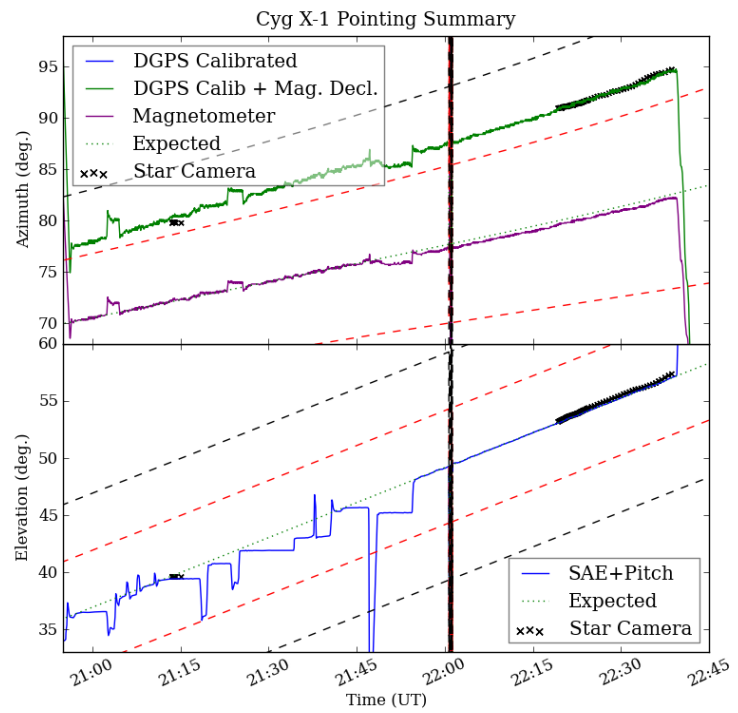

(b)

Figure 5: (a) The temperature and altitude recorded during the flight. The DGPS suffered a catastrophic failure at approximately 15:50 UT (see table 2), nearly coincident with the observation of the minimum external temperature, forcing the aspect system to rely on the backup magnetometer for the determination of the coarse azimuth position. (b) The aspect data taken during the Cyg X-1 observation which was carried out using only the magnetometer and the shaft angle encoder. Instability in the elevation axis, and to a limited extent in the azimuth, can clearly be seen prior to 22:50 UT. Afterward the instability disappeared and stable tracking was maintained until termination of observation at approximately 22:40. The black crosses are the reconstructed aspect solutions for each of the available star camera images retrieved after landing which confirm the validity of the corrected magnetometer aspect solution. The red dashed lines and the black dashed lines show the extent of the fully and partially coded field of view respectively. A post-flight analysis of the aspect data revealed a $\sim 7^{\circ}$ offset from the target position, consistent with the reconstructed position of Cyg X-1 and star camera measurements.

\begin{tabular}{|l|l|}
\hline Time (MDT) & Event \\
\hline $08: 40$ & Launch \\
$09: 50$ & DGPS failure \\
$11: 00$ & Float altitude achieved \\
$11: 30$ & Stow pin removed \\
$11: 40$ & Begin 3C237 observation \\
$12: 25$ & Begin shield threshold testing \\
$12: 55$ & End shield threshold testing \\
$14: 34$ & Begin slew to Cygnus X-1 \\
$15: 00$ & Begin Cygnus X-1 observation \\
$16: 00$ & CIP Card FCB Power Off \\
$16: 03$ & CIP Card FCB Power On \\
$16: 50$ & Flight termination \\
\hline
\end{tabular}

Table 2: A brief overview of the milestones which occured throughout the flight. 


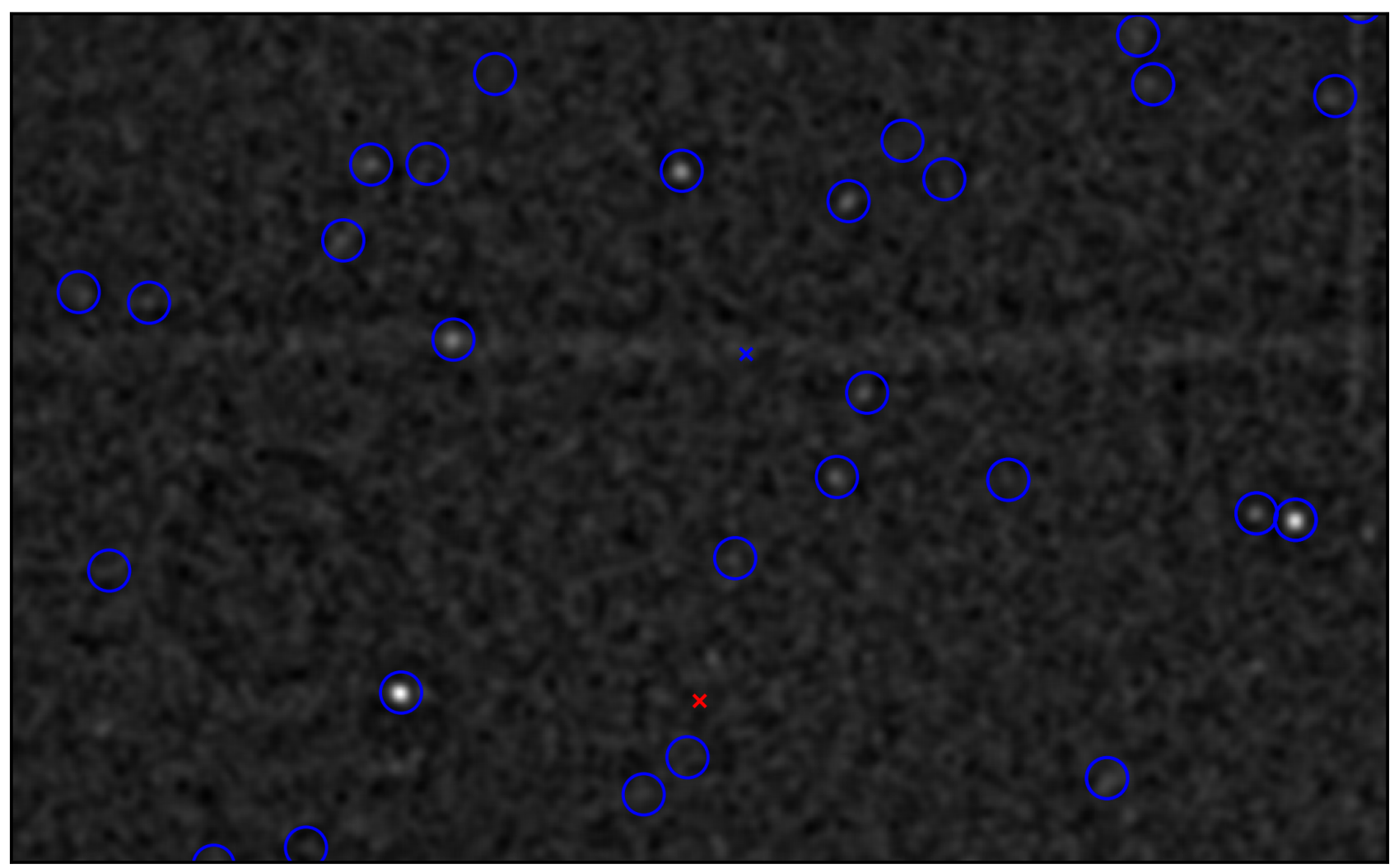

Figure 6: A single star camera image taken at 22:22:19 UT on the $9^{\text {th }}$ of October 2009. In order to extract sources from a single star camera image a large scale background subtraction is applied using a boxcar smoothed image with a $75 \times 75$ pixel kernel as a rough background estimate. A 2D gaussian filter with $\sigma=5$ pixels is then applied and a source list extracted with SExtractor. ${ }^{21}$ The source list is passed to astrometry.net-0.25 ${ }^{22}$ in order to determine an aspect solution for the image. The post processed image is shown above with the detected sources marked with blue circles. The stars detected in this image have a $V_{\text {mag }}$ between approximately 9.5 and 9.0 .

\subsection{Pointing System Performance}

During the flight 3 major difficulties with the pointing system were encountered which complicated flight operations and the analysis of the flight data: the failure of the differential global positioning system (DGPS) shortly after liftoff, the failure of the daytime star camera to acquire an aspect solution during the flight, and sporadic instability in the elevation axis.

During the first half of the flight instability of the elevation axis was a major issue (see figure 5b) and prevented the stable observation of 3C 273. Fortunately stability improved as the flight progressed and a long observation of Cyg X-1 was possible. The suspected cause is a combination of stiff wiring at low temperature in combination with a relatively weak elevation axis motor. In anticipation of future flights the replacement of the elevation axis motor is being considered.

The most serious issue encountered was the loss of the DGPS module during the ascent of the payload (see figure 5a). As the DGPS is the primary method by which an accurate azimuthal position for the coarse pointing system is determined its loss forced the aspect system to fall back on the magnetometer. Unfortunately magnetometers have large intrinsic systematics associated with their orientation relative to the position of other structures on the payload as well as their position on the earth; a post-flight analysis of the magnetometer data revealed substantial systematic offsets of up to $50^{\circ}$. On ascent attitude control was offline and the gondola was allowed to rotate freely, which enabled the collection of simultaneous measurements of the azimuth by both the magnetometer and the DGPS over the entire $360^{\circ}$. These measurements were later used to calibrate the 
magnetometer, which removed the most egregious systematic errors in the azimuth measurement. After the application of an additional correction taking into account the change of the magnetic declination over the course of the flight utilizing GEOMAG IGRF10*, a model of the earth's magnetic field, the pointing data was reconciled with aspect solutions determined from archived star camera images. The cause of the DGPS failure is currently under investigation, however, since the DGPS unit is mounted externally and the failure was nearly coincident with the observation of the minimum recorded external temperature it is highly likely it was the result of insufficient thermal insulation and heaters. To guard against failure in future flights new heating and insulation schemes are under consideration. As an additional safeguard an on-board magnetometer calibration subroutine is also being contemplated to mitigate the large systematic pointing errors in the event of a DGPS failure on the next flight.

The next major difficulty was the failure of the daytime star camera to acquire a pointing solution during the flight. This was primarily the result of bad columns in the star camera CCD, a large offset between the true pointing position and the measure position due to the loss of the DGPS, and to a lesser extent insufficient shielding from the atmospheric background light at certain positions on the sky. The offset of the measured azimuthal value using the magnetometer likely prevented the determination of an aspect solution during the majority of the flight due to the finite star catalog search radius (approximately $4^{\circ}$ ) about the coarse aspect system's reported position. To correct for these problems the CCD has been returned to the manufacturer for repair, an evaluation of the refurbished CCD is planned within the next year. In addition to this alternative star field search algorithms are being considered for the star camera in order to increase the size of the search radius. In spite of these difficulties usable star camera images archived during the flight proved useful in reconstruction of the telescope aspect information. Using cleaned archived star camera images the extraction of star positions in the image using SExtractor ${ }^{21}$ then an aspect solution using astrometry.net- $0.25^{22}$ was possible. Images were cleaned by removal of bad columns from the image and replacement of the bad column pixel values with the average taken from their 6 nearest neighbors, a rough flat fielding and application of a 2D Gaussian filter with $\sigma=5$ pixels was applied to the entire image (see figure 6). After the aspect information for all star camera images was determined an aspect solution was generated then compared to the corrected magnetometer / elevation aspect data. Using this it was possible to independently confirm the validity of the corrected magnetometer aspect solution (see figure 5).

\subsection{Telescope System Performance}

The telescope and the associated subsystems performed without incident during the flight. The science payload heating system and control mechanism maintained a steady internal temperature of $20 \pm 3^{\circ} \mathrm{C}$ for the entire duration of the flight. The flight computer and associated command and control system did not experience a single failure enabling the receipt of data from liftoff through flight termination. The internal power control and monitoring mechanism proved adequate; toward the end of the flight a system test was conduced to confirm the stable operation of the independent CIP interfaced power control mechanism as well as to test the complete remote reinitialization of the detector plane and other subsystems. The restart procedure, save a minor glitch in the data readouts, was a complete success. Within $15 \mathrm{~min}$. after the restoration of power the telescope and all subsystems were running within nominal parameters and celestial observations were resumed.

In order to verify the operation of the coded-aperture telescope an observation of Cyg X-1 and 3C 273 was carried out. As outlined in $\S 5.1$ the observation of $3 \mathrm{C} 273$ proved untenable due to issues encountered with the pointing system, however sufficient pointing stability was achieved during the observation of Cyg X-1. After application of corrected aspect data Cyg X-1 was detected at $7.2 \sigma$ with $\sim 7^{\circ}$ offset after 10 minutes of stable observation at the end of the flight (c.f. Hong et. al. in preparation ${ }^{23}$ ). During the flight the background spectra and detector behavior were also monitored for any deviations in behavior due to charge saturation in the high radiation environment. In spite of the large increase in the background event rate the detector plane operated normally without any degradation in performance. The spectral response of the detector was monitored in flight using the tagged ${ }^{241} \mathrm{Am}$ source and, as was observed on the ground the and during the flight. A slight increase in the average energy resolution was observed, $4.0 \mathrm{keV}$ in flight vs. $3.5 \mathrm{keV}$ in the hanger (FWHM) at $59.6 \mathrm{keV}$ averaged over all active pixels for the duration of the flight. The detector gain remained constant over

\footnotetext{
*http://www.ngdc.noaa.gov/IAGA/vmod
} 

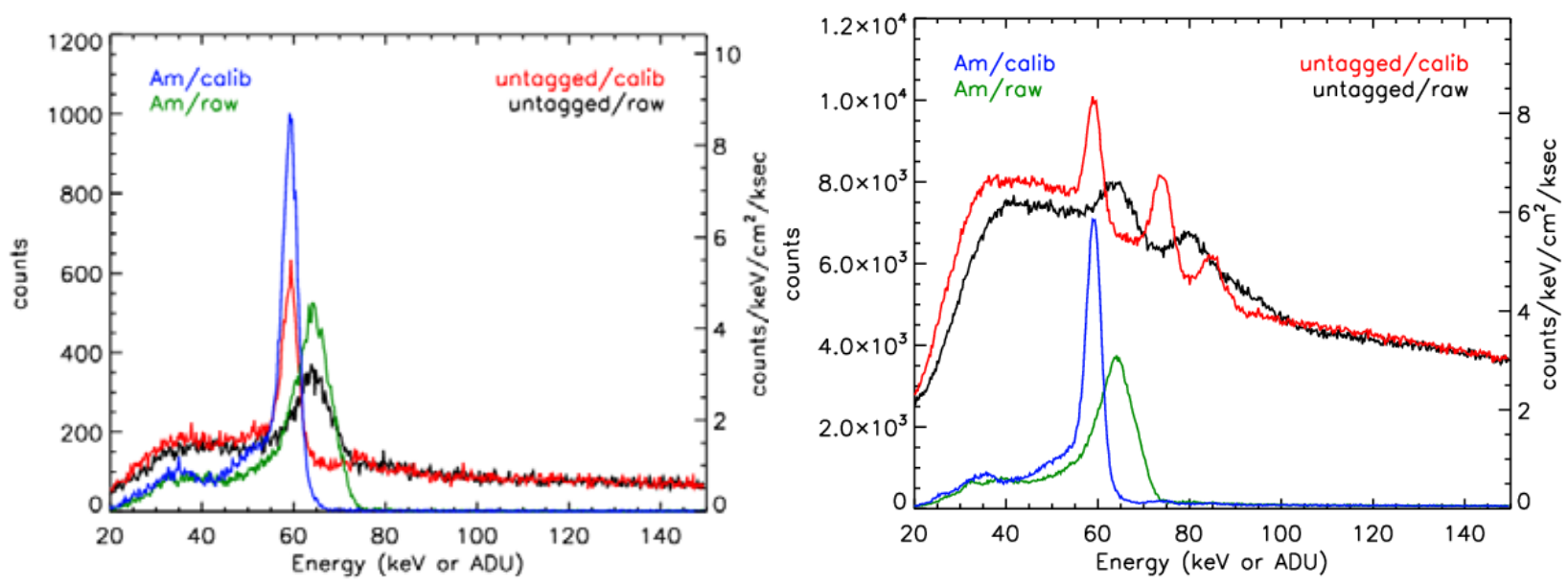

Figure 7: (a) The preflight calibrated and non-calibrated spectra over the entire detector plane for the ${ }^{241} \mathrm{Am}$ calibration source and the background spectrum measured in the hanger at Ft. Sumner, NM. The measured energy resolution after calibration is $3.5 \mathrm{keV}$ FWHM at $59.6 \mathrm{keV}$. (b) Calibrated and uncalibrated, background and calibration spectrum observed in flight. The two additional lines visible above the $59.6 \mathrm{keV}{ }^{241} \mathrm{Am}$ line are the $\mathrm{K} \alpha$ and $\mathrm{K} \beta$ fluorescence lines emitted from the segment of unshielded $\mathrm{Pb}$ on the lower side shield section (see §4.1). The energy resolution measured in flight is approximately $4 \mathrm{keV} \mathrm{FWHM} \mathrm{at} 59.6 \mathrm{keV}$.

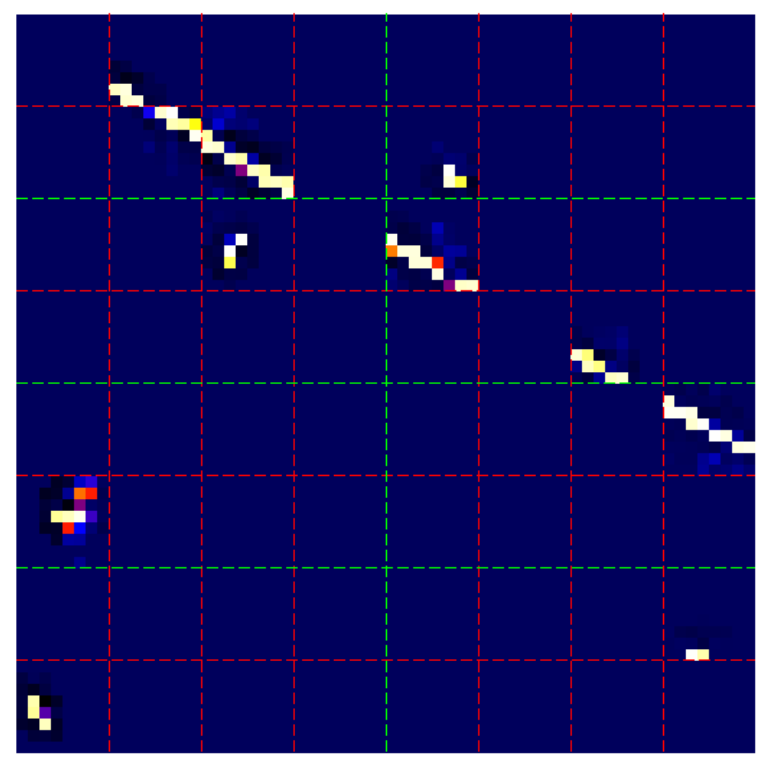

$11 \quad 133$

257

379

503

625

748

871

Figure 8: A detector plane image of the track and charge deposited by a single cosmic ray skimming the top of the detector plane. The breaks in the track are a consequence of the independence of the 32 individual DCU pairs and the $\sim 30 \mu$ s deadtime. This image was generated taking a coincidence window of $200 \mu$ s between events recorded on individual DCU pairs. The color scale is the uncalibrated energy recorded in each detector pixel. The dashed green lines represent the DCA borders, and the dashed red lines represent the borders between DCUs. 


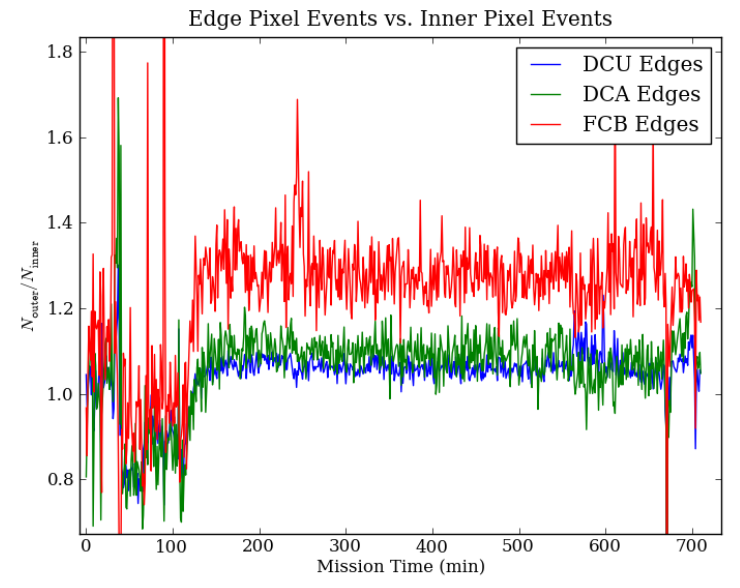

(a)

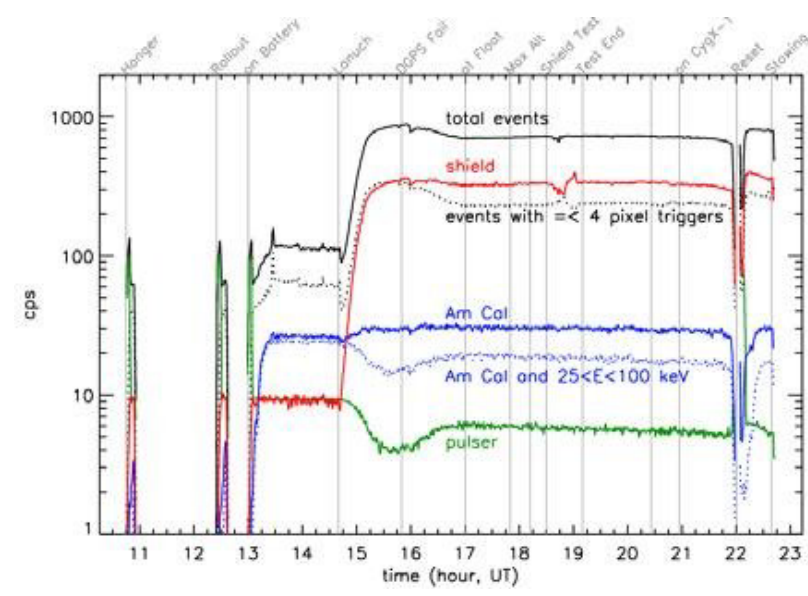

(b)

Figure 9: (a) The ratio of the total background rate for pixels located on the edge of the FCB (red), on the edges between the individual DCAs (green), and on the edges between the individual DCUs (blue), to total the background rate of pixels located within the DCU's. Pixels located on the periphery of the detector plane experienced a $30 \%$ higher background rate than the pixels located in the middle of the DCUs, while those located on the edges adjacent to neighboring detectors only experienced a $5 \%$ increase. (b) The detector rates over the entire flight remained remarkably stable once the payload reached float altitude. The only changes in rate occurred in the shield during the test adjustment of the threshold values (around 18:30 UT) and during reinitialization of the detector system after the power test (22:00 UT) and the required change in threshold values on restart.

the duration of the flight as did the background at $4.0 \times 10^{-3} \mathrm{~cm}^{-2} \mathrm{~s}^{-1} \mathrm{keV}^{-1}$ above $120 \mathrm{keV}$; in line with expectations. Additionally one of the key advantages of the tiled detector configuration proposed for EXIST, namely the reduction of background rates through shielding of the individual detector edges with a neighboring detector, was clearly demonstrated as well. For pixels on the edge of the detector plane, 252 in all, the measured backgrounds were $30 \%$ higher than observed in pixels located in the central (i.e. non-edge) pixels of the DCUs. For edges shielded by an adjacent detector, 1540 pixels total, a $\sim 5 \%$ higher background rate was observed. Extrapolating from these rates had the ProtoEXIST1 detector not been closely tiled fully exposing all DCU edges a $10 \%$ increase in the background rate would be expected (see figure 9 ).

\section{FUTURE TELESCOPE DEVELOPMENT}

Development for EXIST is currently continuing with ProtoEXIST2. ProtoEXIST2 will utilize the DB-ASIC for the readout of $19.5 \mathrm{~mm} \times 19.5 \mathrm{~mm} \times 5.0 \mathrm{~mm}$ CZT detectors with a $32 \times 32$ array of anode pixels with a $600 \mu \mathrm{m}$ pitch. Using a slightly modified version of the ProtoEXIST1 modularization scheme (see $\S 3$ ); a $4 \times 4$ sub-array of these detectors (1/4 of a detector plane) will be fabricated and demonstrated. It is anticipated that testing will begin on the first detector modules for ProtoEXIST2 within the next few months with the completion of the first sub-array occurring before April of 2011. In ProtoEXIST3 the remaining 3/4 of the detector plane will be built using a lower power version derived from the DB-ASIC which consumes about a factor of four less power, the EXIST Final or EX-ASIC. With the completion of the detector plane a test flight with both the original ProtoEXIST1 and new ProtoEXIST2/3 detector planes will be be carried out in the fall of 2012. The ProtoEXIST2/3 telescope will feature an extended $2 \mathrm{~m}$ focal length, be capable of imaging sources with an angular resolution of $2.4^{\prime}$ and have a fully coded $2.3^{\circ} \times 2.3^{\circ}$ field of view. Parallel to this effort a further modification of the EX-ASIC design for the replacement of wirebond pads, which prevent close tiling over the entire detector plane, with microvias to form the final EXIST ASIC (EXF-ASIC). 


\section{CONCLUSIONS AND FUTURE PLANS}

The successful integration of the ProtoEXIST1 detector plane and its operation at high altitude in a near space environment have demonstrated the feasibility of operating a large area tiled detector plane similar to the EXISTHET utilizing individual pixiliated CZT detectors (DCUs) as the core building block. Measured spectra revealed that the high background rates and charge saturation due to incident cosmic rays had little impact $(\sim 10 \%)$ on the detector energy resolution and no discernible impact on the detector gains. The elevated event rates also did not pose any difficulties for the detector plane electronics or the data acquisition system. It has also been demonstrated that the interference between the individual DCUs on the ground and in flight does not pose a significant challenge to the construction of large tiled arrays. In short the operation of the telescope and science package was a complete success on the ground and in flight. Additionally a key advantage of the tiled array, the mutual shielding of detector edges by neighboring DCUs, was also demonstrated in flight showing that a reduction in backgrounds of at least $10 \%$ is possible over a non-tiled detector plane configuration.

In the near future repair of the landing damage on the gondola will be carried out as will improvements to the pointing system to improve reliability. Once the detector integration for ProtoEXIST2/3 is completed a flight together with the original ProtoEXIST1 detector plane is planned for characterization and comparison of both systems in flight.

\section{REFERENCES}

[1] Ubertini, P., Lebrun, F., Di Cocco, G., Bazzano, A., Bird, A. J., Broenstad, K., Goldwurm, A., La Rosa, G., Labanti, C., Laurent, P., Mirabel, I. F., Quadrini, E. M., Ramsey, B., Reglero, V., Sabau, L., Sacco, B., Staubert, R., Vigroux, L., Weisskopf, M. C., and Zdziarski, A. A., "IBIS: The Imager on-board INTEGRAL," Astronomy 83 Astrophysics 411, L131-L139 (Nov. 2003).

[2] Lebrun, F., Leray, J. P., Lavocat, P., Crétolle, J., Arquès, M., Blondel, C., Bonnin, C., Bouère, A., Cara, C., Chaleil, T., Daly, F., Desages, F., Dzitko, H., Horeau, B., Laurent, P., Limousin, O., Mathy, F., Mauguen, V., Meignier, F., Molinié, F., Poindron, E., Rouger, M., Sauvageon, A., and Tourrette, T., "ISGRI: The INTEGRAL Soft Gamma-Ray Imager," Astronomy 83 Astrophysics 411, L141-L148 (Nov. 2003).

[3] Barthelmy, S. D., Barbier, L. M., Cummings, J. R., Fenimore, E. E., Gehrels, N., Hullinger, D., Krimm, H. A., Markwardt, C. B., Palmer, D. M., Parsons, A., Sato, G., Suzuki, M., Takahashi, T., Tashiro, M., and Tueller, J., "The Burst Alert Telescope (BAT) on the SWIFT Midex Mission," Space Science Reviews 120, 143-164 (Oct. 2005).

[4] Barrett, H. H., Eskin, J. D., and Barber, H. B., "Charge Transport in Arrays of Semiconductor Gamma-Ray Detectors," Physical Review Letters 75, 156-159 (July 1995).

[5] Doty, F. P., Barber, H. B., Augustine, F. L., Butler, J. F., Apotovsky, B. A., Young, E. T., and Hamilton, W., "Pixellated CdZnTe detector arrays," Nuclear Instruments and Methods in Physics Research A 353, 356-360 (Dec. 1994).

[6] Harrison, F. A., Christensen, F. E., Craig, W., Hailey, C., Baumgartner, W., Chen, C. M. H., Chonko, J., Cook, W. R., Koglin, J., Madsen, K., Pivavoroff, M., Boggs, S., and Smith, D., "Development of the HEFT and NuSTAR focusing telescopes," Experimental Astronomy 20, 131-137 (Dec. 2005).

[7] Baumgartner, W. H., Tueller, J., Krimm, H., Barthelmy, S. D., Berendse, F., Ryan, L., Birsa, F. B., Okajima, T., Kunieda, H., Ogasaka, Y., Tawara, Y., and Tamura, K., "InFOCuS hard x-ray telescope: pixellated CZT detector/shield performance and flight results," in [Society of Photo-Optical Instrumentation Engineers (SPIE) Conference Series], J. E. Truemper \& H. D. Tananbaum, ed., Society of Photo-Optical Instrumentation Engineers (SPIE) Conference Series 4851, 945-956 (Mar. 2003).

[8] Hong, J., Grindlay, J. E., Allen, B., Barthelmy, S. D., Skinner, G. K., and Gehrels, N., "The high energy telescope on EXIST," in [Society of Photo-Optical Instrumentation Engineers (SPIE) Conference Series], Society of Photo-Optical Instrumentation Engineers (SPIE) Conference Series 7435 (Aug. 2009).

[9] Hong, J. et. al.., "The proposed high-energy telescope (het) for exist," These Proceedings $\mathbf{7 7 3 2 .}$

[10] Rana, V. R., Cook, III, W. R., Harrison, F. A., Mao, P. H., and Miyasaka, H., "Development of focal plane detectors for the Nuclear Spectroscopic Telescope Array (NuSTAR) mission," in [Society of Photo-Optical Instrumentation Engineers (SPIE) Conference Series], Society of Photo-Optical Instrumentation Engineers (SPIE) Conference Series $\mathbf{7 4 3 5}$ (Aug. 2009). 
[11] Grindlay, J. E., Garcia, M. R., Burg, R. I., and Murray, S. S., "The energetic X-ray imaging telescope experiment (EXITE)," IEEE Transactions on Nuclear Science 33, 750-754 (Feb. 1986).

[12] Ramsey, B. D., Alexander, C. D., Apple, J. A., Benson, C. M., Dietz, K. L., Elsner, R. F., Engelhaupt, D. E., Ghosh, K. K., Kolodziejczak, J. J., O’Dell, S. L., Speegle, C. O., Swartz, D. A., and Weisskopf, M. C., "First Images from HERO, a Hard X-Ray Focusing Telescope," Astrophysical Journal 568, 432-435 (Mar. 2002).

[13] Dietz, K. L., Ramsey, B. D., Alexander, C. D., Apple, J. A., Ghosh, K., and Swift, W. R., "Daytime aspect camera for balloon altitudes," Optical Engineering 41, 2641-2651 (Oct. 2002).

[14] Hong, J., Allen, B., Grindlay, J., Chammas, N., Barthelemy, S., Baker, R., Gehrels, N., Nelson, K. E., Labov, S., Collins, J., Cook, W. R., McLean, R., and Harrison, F., "Building large area CZT imaging detectors for a wide-field hard X-ray telescope-ProtoEXIST1," Nuclear Instruments and Methods in Physics Research A 605, 364-373 (July 2009).

[15] Hong, J., Grindlay, J. E., Chammas, N., Allen, B., Copete, A., Said, B., Burke, M., Howell, J., Gauron, T., Baker, R. G., Barthelmy, S. D., Sheikh, S., Gehrels, N., Cook, W. R., Burnham, J. A., Harrison, F. A., Collins, J., Labov, S., Garson, III, A., and Krawczynski, H., "Wide-field hard x-ray survey telescope: ProtoEXIST1," in [Society of Photo-Optical Instrumentation Engineers (SPIE) Conference Series], Presented at the Society of Photo-Optical Instrumentation Engineers (SPIE) Conference 6706 (Sept. 2007).

[16] Hong, J., Grindlay, J. E., Chammas, N., Copete, A., Baker, R. G., Barthelmy, S. D., Gehrels, N., Cook, III, W. R., Burnham, J. A., Harrison, F. A., Collins, J., and Craig, W. W., "CZT imaging detectors for ProtoEXIST," in [Society of Photo-Optical Instrumentation Engineers (SPIE) Conference Series], Presented at the Society of Photo-Optical Instrumentation Engineers (SPIE) Conference 6319 (Aug. 2006).

[17] Allen, B. et. al.., "The ProtoEXIST1 Coded-Aperture Hard X-Ray Telescope," In Preparation (2010).

[18] Robinson, D. W., "Building a new kind of graded-Z shield for Swift's burst alert telescope," in [Society of Photo-Optical Instrumentation Engineers (SPIE) Conference Series], J. E. Truemper \& H. D. Tananbaum, ed., Presented at the Society of Photo-Optical Instrumentation Engineers (SPIE) Conference 4851, 13741381 (Mar. 2003).

[19] Fenimore, E. E. and Cannon, T. M., "Coded aperture imaging with uniformly redundant arrays," Applied Optics 17, 337-347 (Feb. 1978).

[20] Busboom, A., Elders-Boll, H., and Schotten, H. D., "Uniformly Redundant Arrays," Experimental Astronomy 8, 97-123 (1998).

[21] Bertin, E. and Arnouts, S., "SExtractor: Software for source extraction.," Astronomy \& Astrophysics Suppliment Series 117, 393-404 (June 1996).

[22] Lang, D., Hogg, D. W., Mierle, K., Blanton, M., and Roweis, S., "Astrometry.net: Blind Astrometric Calibration of Arbitrary Astronomical Images," Astronomical Journal 139, 1782-1800 (May 2010).

[23] Hong, J. et. al.., "Flight performance of an advanced czt imaging detector in a wide-field hard x-ray telescope- protoexist1," In Preparation (2010). 